\title{
Understanding the doping dependence of the conductivity of conjugated polymers: dominant role of the increasing density of states and growing delocalization
}

Citation for published version (APA):

Martens, H. C. F., Hulea, I. N., Romijn, I. G., Brom, H. B., Pasveer, W. F., \& Michels, M. A. J. (2003).

Understanding the doping dependence of the conductivity of conjugated polymers: dominant role of the increasing density of states and growing delocalization. Physical Review B, 67(12), 121203-1/4. [121203]. https://doi.org/10.1103/PhysRevB.67.121203

DOI:

10.1103/PhysRevB.67.121203

Document status and date:

Published: 01/01/2003

Document Version:

Publisher's PDF, also known as Version of Record (includes final page, issue and volume numbers)

\section{Please check the document version of this publication:}

- A submitted manuscript is the version of the article upon submission and before peer-review. There can be important differences between the submitted version and the official published version of record. People interested in the research are advised to contact the author for the final version of the publication, or visit the $\mathrm{DOI}$ to the publisher's website.

- The final author version and the galley proof are versions of the publication after peer review.

- The final published version features the final layout of the paper including the volume, issue and page numbers.

Link to publication

\footnotetext{
General rights

- You may freely distribute the URL identifying the publication in the public portal. follow below link for the End User Agreement:

www.tue.nl/taverne

\section{Take down policy}

If you believe that this document breaches copyright please contact us at:

openaccess@tue.nl

providing details and we will investigate your claim.
}

Copyright and moral rights for the publications made accessible in the public portal are retained by the authors and/or other copyright owners and it is a condition of accessing publications that users recognise and abide by the legal requirements associated with these rights.

- Users may download and print one copy of any publication from the public portal for the purpose of private study or research.

- You may not further distribute the material or use it for any profit-making activity or commercial gain

If the publication is distributed under the terms of Article $25 \mathrm{fa}$ of the Dutch Copyright Act, indicated by the "Taverne" license above, please 


\title{
Understanding the doping dependence of the conductivity of conjugated polymers: Dominant role of the increasing density of states and growing delocalization
}

\author{
H. C. F. Martens, ${ }^{1}$ I. N. Hulea, ${ }^{1,3}$ I. Romijn, ${ }^{1}$ H. B. Brom, ${ }^{1}$ W. F. Pasveer ${ }^{2,3}$ and M. A. J. Michels ${ }^{2,3}$ \\ ${ }^{1}$ Kamerlingh Onnes Laboratory, Leiden University, P.O. Box 9504, 2300 RA Leiden, The Netherlands \\ ${ }^{2}$ Eindhoven University of Technology, P.O. Box 513, NL-5600 MB Eindhoven, The Netherlands \\ ${ }^{3}$ Dutch Polymer Institute, P.O. Box 902, NL-5600 AX Eindhoven, The Netherlands
}

(Received 9 October 2002; revised manuscript received 26 November 2002; published 10 March 2003)

\begin{abstract}
In variable-range-hopping theories for the dc conductivity, the extension of sites where the charges are located and the energy dependence of the density of states (DOS) are usually neglected. We show that these dependences are the dominant factors for understanding the strong doping dependence, and present an analytical theory for arbitrary DOS. We verify the theory with systematic data over a broad range of temperature and doping for $\mathrm{FeCl}_{3}$-doped poly( $p$-phenylene vinylene). By combining theory and data, we reconstruct the energy-dependent DOS and the extension of sites.
\end{abstract}

DOI: 10.1103/PhysRevB.67.121203

PACS number(s): 72.80.Le, 71.20.Rv, 72.20.Ee, 73.61.Ph

The strong increase in conductivity with doping of conjugated polymers was the first essential breakthrough that made the field of polymer electronics the revolutionary field that it is today. Although the fact has been demonstrated and discussed many times since, ${ }^{1}$ the steeply nonlinear functional relation between dopant concentration and conductivity in the insulating state has not been consistently explained. ${ }^{2-5}$ For the more frequently studied and equally steep temperature dependence of the conductivity, ${ }^{5-8}$ various approaches exist, which have in common that some kind of phononassisted hopping between localized states allows the dc transport. In these models disorder, correlations and (bi)polaron formation can play a role as well and often simplifying assumptions have been made about the shape of the density of states (DOS). ${ }^{1,5,9-13}$ Because of these differences, it will be helpful to follow a more general approach, the results of which might also guide more detailed microscopic descriptions. Here we start from Mott's three-dimensional (3D) variable range hopping (VRH) and allow for a spatial extension of the localized states that were originally approximated as dimensionless points. It appears that without specifying the exact nature of the charge carriers involved in the conductivity, we can clarify the relation between conductivity and doping level in the nonmetallic state for the common case, where 3D charge transport prevails already at the lowest doping levels. ${ }^{14}$ The major outcomes are as follows.

(1) A fully general analytical result [see Eq. (4)], identifying how the strong dopant dependence relates to two dominant effects: the increasing DOS at higher concentrations, and the growth of the delocalized regions. This result not only gives mechanistic insight that was so far absent, but is mathematically applicable to any shape of the DOS.

(2) An extension [see Eq. (5)], accounting for the dopant/ delocalization dependence, of the classical result of Mott, which has so far been the reference for modeling the $T$-dependent variable-range-hopping conductivity in doped polymers.

(3) Successful verification of the theory with very precise and systematic data over a broad range of concentration and temperature, using $\mathrm{FeCl}_{3}$-doped poly( $p$-phenylene vinylene) (PPV) as paradigm.
(4) Actual reconstruction, from the combined theory and data, of the energy-dependent DOS of the doped polymer. Although these results are derived for the insulating state, we expect them to be relevant for our understanding of the metal-insulator transition and the metallic phase of these conjugated polymers as well.

$\mathrm{OC}_{1} \mathrm{C}_{10}$-PPV was doped in solution with iron(III)chloride, $\mathrm{FeCl}_{3}$. Ideally, the following redox reaction should take place: $\mathrm{PPV}+2 \mathrm{FeCl}_{3} \rightarrow \mathrm{PPV}^{+}+\mathrm{FeCl}_{2}+\mathrm{FeCl}_{4}^{-}$. The doping level $c$ is defined as the number of carriers per monomer. ${ }^{15}$ We refer for more details to Ref. 14. Under ambient conditions, the conductive properties of the films are stable over several weeks.

The $T$ dependence of $\sigma$ is presented in Fig. 1, where $\sigma$ is plotted versus $T^{-1 / 4}$ on a $\log$-linear scale. At sufficiently low $T$, Mott's law $\sigma(T)=\sigma_{0} \exp \left[-\left(T_{0} / T\right)^{1 / 4}\right]$ for threedimensional VRH holds. ${ }^{16}$ Such a $T$ dependence of $\sigma$ has been frequently reported in doped conjugated polymers, ${ }^{5-8}$ and is suggestive of thermally activated tunneling of carriers between localized states in a constant density of states. As in other conjugated polymers, ${ }^{2-5} \sigma$ increases steeply with doping level: by increasing $c$ from 0.005 to $0.2, \sigma$ increases by

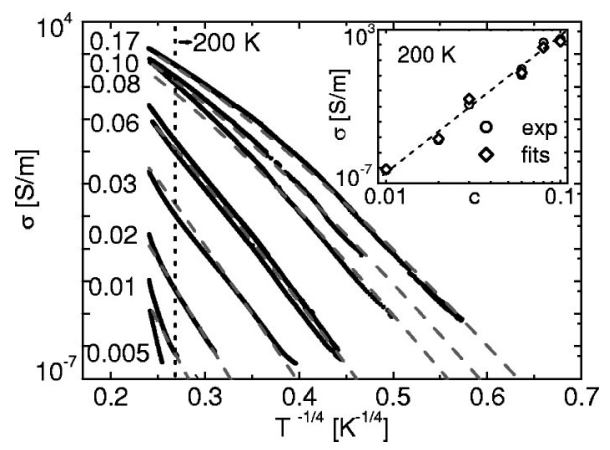

FIG. 1. (a) $\sigma$ vs $T^{-1 / 4}$ for $0.005<c<0.17$ (two samples have $c$ close to 0.06). Doping levels are expressed per monomer. At low $T$, Mott's law $\sigma(T)=\sigma_{0} \exp \left[-\left(T_{0} / T\right)^{1 / 4}\right]$ for three-dimensional VRH holds. Dashed lines are fits discussed in the text. Inset shows the powerlike dependence of $\sigma$ for $0.01 \leqslant c \leqslant 0.1$ at $200 \mathrm{~K}: \sigma \propto c^{8}$ (dashed). 


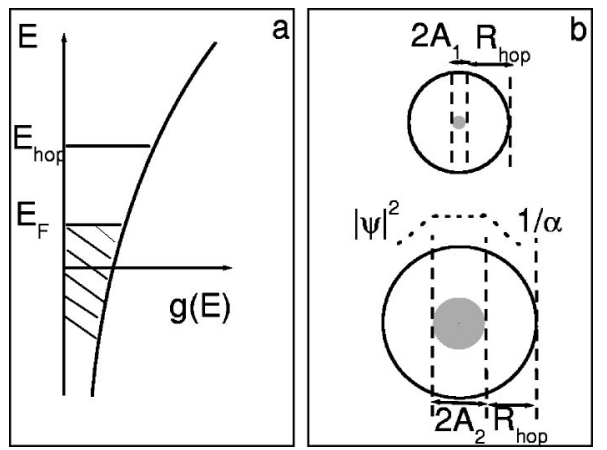

FIG. 2. (a) $E$ vs $g(E)$. States within $E_{\text {hop }}$ and $E_{F}(c)$ are available for hopping (b) $A(c)$. With increasing extent $A$ of the localized states the number of available states, within a given $R_{\text {hop }}$ increases. The typical decay length of the electron density $\psi^{2}$ is $1 / \alpha$.

seven orders of magnitude. At high $T$, deviations from Mott's law occur: the low-c samples reveal a stronger, and the high$c$ samples a weaker $T$ dependence of $\sigma$, see Fig. 1 .

Qualitatively, the strong $c$ dependence of $\sigma$ can be understood as follows. Due to disorder and self-trapping (polarons), the electronic states are spatially localized and distributed in energy $(E)$. Charge transport occurs by means of VRH. Two contributions to $\sigma(c)$ can be considered. First, in conjugated polymers the density of (localized) states $g(E)$ is energy dependent as schematically indicated in Fig. 2(a). ${ }^{9,12,17}$ When filling such a DOS, more states within a given hopping energy $\epsilon=E_{\text {hop }}-E_{F}(c)$ become available and this enhances $\sigma$ exponentially. Also doping-induced states near $E_{F}$ result in a similar increase of $\sigma$. Second, the size of the localized regions (at least a monomer) may grow with increasing $c$ (and thus $E_{F}$ ) as well. The rationale behind this is that states at high $E$ are less strongly bound by the random potential, i.e., are spatially more extended. ${ }^{18}$ As the number of states within a given hopping distance $R$ from these finitesize localized sites increases with the localization volume, see Fig. 2(b), $\sigma$ increases strongly as well. Note that $\sigma$ of the high- $c$ samples is only an order of magnitude below Mott's minimum metallic conductivity at high $T$, i.e., a point model of the localized states cannot be expected to hold.

For the peculiar $T$ dependence of $\sigma$, we have to keep in mind that at low $c$ the hopping energy is relatively large since $g\left(E_{F}\right)$ is low. If $g\left(E_{\mathrm{hop}}\right)>g\left(E_{F}\right)$, more states are available for the hopping process than expected for constant $g$ and also nearest-neighbor hops become more important: $\sigma$ will be activated (e.g., for $c=0.01$ the activation energy is around $0.5 \mathrm{eV})$. At high $c, g\left(E_{F}\right)$ is larger resulting in smaller hopping distances, and thus the nonzero extent of the localized states comes into play: within a given hopping distance more states are available and the hop activation energy will decrease. This explains the weaker $T$ dependence of $\sigma$ observed at high $T$ and high $c$.

We now derive a VRH expression for $\sigma(c)$ in a system with an arbitrary shape of $g(E)$ and volume $V_{0}$ of the localized region. We consider a carrier located at the Fermi level. The squared wave function decays within a length $1 / \alpha$ (in point-site models $1 / \alpha \gg A$, and $L=1 / \alpha$ is referred to as the localization length). The hopping probability depends expo- nentially both on the hopping distance $R$ between the localized regions and the activation energy $\left(E-E_{F}\right) .{ }^{19}$ The density of final sites $\mathcal{N}$ that can be reached with activation energy less than $E-E_{F}$ is given by $\mathcal{N}\left(E, E_{F}\right)$ $=\int_{E_{F}}^{E} g\left(E^{\prime}\right) d E^{\prime}$. Mott's criterion states that $\sigma$ is governed by those hops for which $E$ and $R$ are such that about one state lies within a volume $V$, defined by: $V \mathcal{N} \approx 1$ [hence $V$ $\left.=V\left(E, E_{F}\right)\right] \cdot{ }^{16}$ For the moment, $R$ is a unique function, to be specified later, of $V$ and of some length $A: R(V, A)$ $=R\left(E, E_{F}, A\right)$. The conductivity can then be written as ${ }^{16,20}$

$$
\sigma=\sigma_{0} \exp \left[-\alpha \widetilde{R}-\beta\left(\widetilde{E}-E_{F}\right)\right]
$$

with $\beta=1 /\left(k_{B} T\right)$, and $\sigma_{0}$ a prefactor. The optimal hop energy $\widetilde{E}$ and associated hop distance $\widetilde{R}$ is obtained if $\alpha R$ $+\beta\left(E-E_{F}\right)$ is minimal or $\alpha(\partial R / \partial E)_{E_{F}, A}+\beta=0$ for $E$ $=\widetilde{E}$. Differentiation of $V \mathcal{N} \approx 1$ to $E$ gives $(\partial / \partial E)_{E_{F}, A} V \mathcal{N}$ $=0$. The condition for $\widetilde{E}$ then becomes

$$
\frac{1}{V^{2}}(\partial V / \partial R)_{A}=\frac{\alpha}{\beta} g(E)
$$

for $E=\widetilde{E}$. These equations establish the optimal hopping energy $\widetilde{E}=E\left(\beta, E_{F}, A\right)$ and hopping distance $\widetilde{R}$ $=R\left[\widetilde{E}\left(\beta, E_{F}, A\right), E_{F}, A\right]$, which yield the system's conductivity according to Eq. (1). The conductivity depends on $c$ via $E_{F}, A$, and $\sigma_{0}$.

To get more insight into these relations, let us study the dependence on $c$ via $E_{F}$ and $A$ explicitly.

$$
\begin{aligned}
\left(\frac{\partial \ln \left(\sigma / \sigma_{0}\right)}{\partial c}\right)_{\beta}= & \left(\frac{\partial \ln \left(\sigma / \sigma_{0}\right)}{\partial E_{F}}\right)_{\beta, A} \frac{d E_{F}}{d c} \\
& +\left(\frac{\partial \ln \left(\sigma / \sigma_{0}\right)}{\partial A}\right)_{\beta, E_{F}} \frac{d A}{d c} .
\end{aligned}
$$

Using Eq. (1), at optimum the first term on the right can be rewritten as $-\alpha\left(\partial \widetilde{R} / \partial E_{F}\right)_{\beta, A}-\beta\left(\partial \widetilde{E} / \partial E_{F}\right)_{\beta, A}+\beta$ $=-\alpha\left(\partial \widetilde{R} / \partial E_{F}\right)_{\widetilde{E}, A}-\alpha(\partial \widetilde{R} / \partial \widetilde{E})_{E_{F}, A}\left(\partial \widetilde{E} / \partial E_{F}\right)_{\beta, A}-\beta(\partial \widetilde{E} /$ $\left.\partial E_{F}\right)_{\beta, A}+\beta=-\alpha\left(\partial \widetilde{R} / \partial E_{F}\right)_{\tilde{E}, A}+\beta=\beta\left[1-g\left(E_{F}\right) / g(\widetilde{E})\right] .{ }^{21}$ In the last step we used the explicit dependence of $\widetilde{R}$ on $E_{F}$ via $\widetilde{V}\left(\widetilde{E}, E_{F}\right)$, which leads to $\left(\partial \widetilde{R} / \partial E_{F}\right) \widetilde{E}, A=\widetilde{V}^{2} g\left(E_{F}\right)(\partial \widetilde{R} /$ $\partial V)_{A}=(\beta / \alpha) g\left(E_{F}\right) / g(E)$. Following similar arguments, we rewrite the second term as $-\alpha(\partial \widetilde{R} / \partial A)_{\beta, E_{F}}-\beta(\partial \widetilde{E} /$ $\partial A)_{\beta, E_{F}}=-\alpha(\partial \widetilde{R} / \partial A)_{\tilde{E}, E_{F}}-\alpha(\partial \widetilde{R} / \partial \widetilde{E})_{A, E_{F}}(\partial \widetilde{E} / \partial A)_{\beta, E_{F}}$ $-\beta(\partial \widetilde{E} / \partial A)_{\beta, E_{F}}=-\alpha(\partial \widetilde{R} / \partial A)_{\widetilde{E}, E_{F}}=\alpha\left[1-V_{0}^{\prime}(A) / V_{0}^{\prime}(A\right.$ $+\widetilde{R})]$. For the last, line we have to realize that $\widetilde{R}$ is a function of $\widetilde{V}\left(\widetilde{E}, E_{F}\right)$ and $A$ only, and hence $(\partial R /$ $\partial A)_{V}(\partial A / \partial V)_{R}(\partial V / \partial R)_{A}=-1$. We also made a more specific assumption $V=V_{0}(A+R)-V_{0}(A)$, still with arbitrary $V_{0}$, see above. The final expression for the dependence of $\sigma / \sigma_{0}$ on $c$ then becomes 


$$
\begin{aligned}
\left(\frac{\partial \ln \left(\sigma / \sigma_{0}\right)}{\partial c}\right)_{\beta}= & \beta\left[\frac{1}{g\left(E_{F}\right)}-\frac{1}{g(\widetilde{E})}\right] \\
& +\alpha\left[\frac{1}{V_{0}^{\prime}(A)}-\frac{1}{V_{0}^{\prime}(A+\widetilde{R})}\right] \frac{d A}{d c},
\end{aligned}
$$

where we replaced $d E_{F} / d c$ in Eq. (3) by $1 / g\left(E_{F}\right)$. This expression is particularly suited to illustrate the $c$ dependence of $\sigma$. In the limit of low doping $\left[A \ll R\right.$, hence $V_{0}^{\prime}(A$ $\left.+\widetilde{R}) \gg V_{0}^{\prime}(A)\right]$, the last term reduces to $\alpha(d A / d c)$ or $\sigma$ $\propto \exp [\alpha A(c)]$. At sufficiently low temperature, we can replace the difference $g\left(E_{F}\right)-g(\widetilde{E})$ by $\left(\widetilde{E}-E_{F}\right) g^{\prime}\left(E_{F}\right)$ which can be neglected. It means that Mott's formula is recovered:

$$
\sigma=\sigma_{0}(c) e^{\alpha A(c)} e^{-\left[T_{0}(c) / T\right]^{1 / 4}}
$$

with the important addition of the factor $e^{\alpha A(c)}$ : At a given temperature, the growth of the localized regime determines for an appreciable part the increase in conductivity. (In the limit that $V_{0}(A)$ can be neglected compared to $V_{0}(A+R)$, the exponential prefactor of Eq. (5) also directly follows from the usual Mott argument, if we replace the hopping variable $R$ by $A+R$. Outside the localized region the wave function $\psi$ [see Fig. 2(b)] decays similarly, but inside it grows with the same exponential factor as added in Eq. (5)]. A power-law dependence of $\sigma$ on $c$, as is often suggested (and also found in our data, where around $200 \mathrm{~K} \sigma \propto c^{p}$ with $p$ around 8, see Fig. 1), then implies a logarithmic dependence of $A$ on $c$. If the first term in Eq. (4) dominates, expected for $g\left(E_{F}\right) \ll g(E)$ relevant for a Gaussian or exponentially growing density of states, ${ }^{9,12} \partial c / \partial \ln \left(\sigma / \sigma_{0}\right)$ is a direct measure of $g\left(E_{F}\right)$.

We now apply this model to reconstruct the $c$ dependence of $A$ and the density of states as function $E_{F}$ for the $\mathrm{FeCl}_{3}$-doped PPV. To obtain an analytic solution, we assume a (nearly) constant density of states $\mathcal{N}\left(E, E_{F}\right) \approx\left(E-E_{F}\right) g$ and spherically symmetric volumes $V=V_{0}(A+R)-V_{0}(A)$ with $V_{0}(A)=(4 \pi / 3) A^{3}$. Under these conditions, $V \mathcal{N} \approx 1$ gives $\left(E-E_{F}\right)=(A+\widetilde{R})(\alpha / 4 \pi g \beta)^{0.5}$, while Mott's criterion leads to $\left(\widetilde{E}-E_{F}\right)=3 /\left[4 \pi g\left(\widetilde{R}^{3}+A^{3}+3 A^{2} \widetilde{R}+3 A \widetilde{R}^{2}\right)\right]$. Subtracting these two equations gives $\widetilde{R}$ and by substitution of $\widetilde{R}$ in $\widetilde{E}-E_{F}$ we obtain $\sigma$ from Eq. (1). ${ }^{22}$ The fits shown in Fig. 1 are based on this solution, which for an appreciable range in $c$ (the lower values) and $T$ (lower temperatures) has to coincide with the predictions of Eq. (5).

Figure 3(a) shows $g\left(E_{F}\right)$ as function of $c$ according to Eq. (5) with $k_{B} T_{0} \propto \alpha^{3} / g .{ }^{23}$ The $T_{0}$ 's are obtained from the lowtemperature tails in Fig. 1. The values for $c=0.01,0.02$, $0.03,0.06,0.08,0.10$, and 0.17 are, respectively, 1.8 $\times 10^{8} \mathrm{~K}, \quad 1.2 \times 10^{8} \mathrm{~K}, \quad 7.5 \times 10^{7} \mathrm{~K}, \quad 5.4 \times 10^{7} \mathrm{~K}, \quad 3.3$ $\times 10^{7} \mathrm{~K}, 3 \times 10^{7} \mathrm{~K}$, and $2.4 \times 10^{7} \mathrm{~K}$, while a rough estimate at $c=0.005$ gives a value of the order of $2.5 \times 10^{8}$. Absolute values of $g(E)$ require an estimate of $\alpha^{-1}$, which is taken as $0.2 \mathrm{~nm},{ }^{24}$ the volume of a monomer $\left(1 \mathrm{~nm}^{3}\right)$ and the proportionality constant (here we take 12 as will be justified later). With these values, $T_{0}$ of $10^{8} \mathrm{~K}$ corresponds to $g \approx 1$ $\times 10^{44}$ states $/ \mathrm{m}^{3} \mathrm{~J}$. This converts to $g \approx 2 \times 10^{-2}$ states/
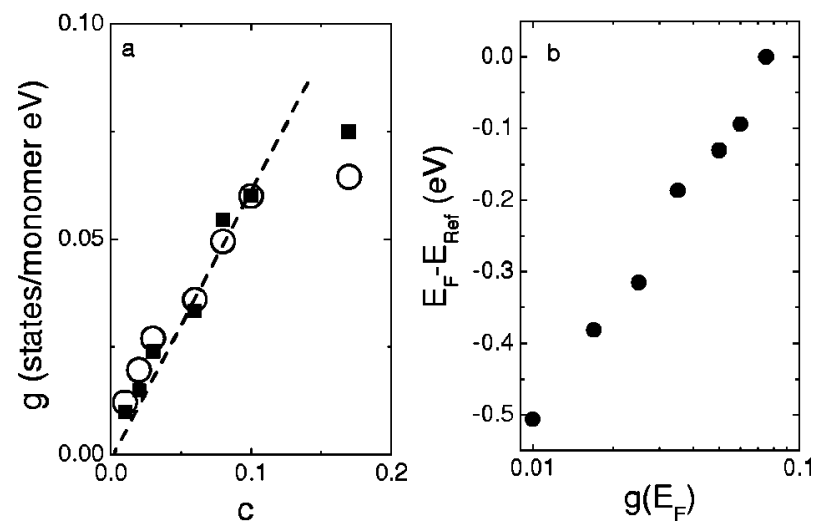

FIG. 3. (a) $g\left(E_{F}\right)$ as a function of doping level. $g\left(E_{F}\right)$ is calculated from Eq. (5) (open circles) and from the model discussed in the text (closed squares). (b) $\left(E_{F}-E_{\text {Ref }}\right)$ vs $g\left(E_{F}\right)$. Data are scaled to reproduce the activation energy of $0.5 \mathrm{eV}$ deduced from the high$T$ data for $c=0.01$, see Fig. 1 .

eV monomer. $g\left(E_{F}\right)$ values from $T_{0}$ (squares) and the fits to the full expression given above (circles) agree when we adjust the proportionality constant to 12 , which compares well with theoretical estimates. ${ }^{23}$ The found dependence of $g\left(E_{F}\right)$ on $c$ is almost linear; the drawn line in Fig. 3(a) corresponds to $g\left(E_{F}, c\right)=0.6 c$. The energy density of states per monomer $g_{m}$ as function of $E_{F}$ [see Fig. 3(b)] can be calculated using $E_{\mathrm{Ref}}-E_{F}=-\int_{c_{\mathrm{Ref}}}^{c}\left[1 / g_{m}(c)\right] d c$, which follows from $d E_{F} / d c=1 / g_{m}\left(E_{F}\right)$. The values found are too high to be realistic (order of $\mathrm{eV}$ ), which is not surprising in view of the crude assumptions made (especially the value of $\alpha$ enters strongly). We scaled the energy scale [vertical axis in Fig. $3(\mathrm{~b})$ ] to reproduce the activation energy of $0.5 \mathrm{eV}$ found from the high- $T$ data of $c=0.01$, see Fig. 1 . Note that an increase of $g$ with $c$ might also result if dopants introduce additional sites or energy states to which hopping can occur. If so, the reconstructed dependence of $g(E)$ on $c$, shown in Fig. 3(a), remains valid, but the plot of $E$ versus $g\left(E_{F}\right)$ loses its validity as it requires $g(E)$ to be $c$ independent.

Although the almost linear increase of $g\left(E_{F}\right)$ with $c$ explains the $T$ dependence at low temperature, it cannot explain the strong $c$ dependence of the data or the deviations at higher temperature. For that purpose, not only the increasing values of $g(E)$ but also of $A$ with $c$ have to be taken into account. Also $\sigma_{0}$ is expected to depend slightly on $c$, e.g. for a flat density of states $\sigma_{0} \propto g\left(E, E_{F}\right)^{1 / 2} \cdot{ }^{23}$ Here we took its value constant $\left(\sigma_{0}=2 \times 10^{5} \mathrm{~S} / \mathrm{m}\right)$. The $A$ values, see Fig. 4(a), range between 1 and $5 \mathrm{~nm}$ and follow the logarithmic dependence expected from the power-law behavior seen in the inset of Fig. 1. Using voltage modulated millimeter-wave spectroscopy on light emitting diodes of chemically undoped PPV, a similar localization volume was found. ${ }^{25}$ Within the model, a saturation of $A$ at high doping levels is not surprising as the localized regions start to consume the total available volume of the polymer. At low doping levels, the saturation length is set by the conjugation length of at least a few monomers along the chain and a monomer in the perpendicular direction; because anisotropy is not included in this simple model, the effective size has to exceed that of a 

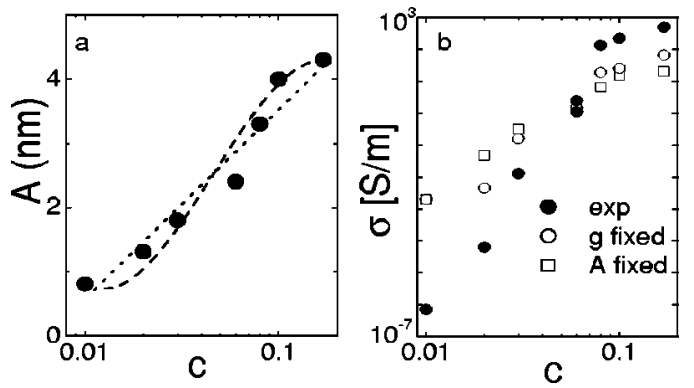

FIG. 4. (a) $A$ (c) from the fits in Fig. 1 with $\sigma_{0}=2 \times 10^{5} \mathrm{~S} / \mathrm{m}$ [for $g(c)$, see Fig. 3]. The dashed line accounts for the saturation at low- and high- $c$ values; the dotted line is the logarithmic dependence, expected from the power-law behavior in the inset of Fig. 1. (b) The contributions to $\sigma(c)$ from the $c$ dependence of $A$ with fixed $g$ and $g$ with fixed $A$ at $200 \mathrm{~K}$.

monomer. In Fig. 4(b), we compare the contributions of $g(c)$ and $A(c)$ to $\sigma(c)$ for $T=200 \mathrm{~K}$, where we kept the values at $c=0.06$ as turning point: the $\sigma(c)$ values were calculated using the calculated values of $g(A)$ with $A(g)$ fixed to 3.5 $\mathrm{nm}\left(g=2.410^{44} / \mathrm{m}^{3} \mathrm{~J}\right)$. Both contributions appear to have a similar effect.

In short, we have shown that for an explanation of the $c$ and $T$ dependence of $\sigma$ in conjugated polymers such as PPV, it is essential to take the $c$ dependence of both $g(E)$ and $A$ into account. The derived equations are valid for an arbitrary DOS. For a quantitative description of the data set, which covers a wide range in $c$ and $T$, Eq. (5) is a good starting point. The growing values of $A$ and $g$ contribute equally to the increase by about eight orders of magnitude in $\sigma$ if $c$ changes from 0.01 to 0.1 . These results are not only important from a fundamental point of view, but for applications dealing with highly conducting polymers as well. It still remains a challenge to relate the extracted parameters to a specific microscopic model for the conduction in these systems (e.g., metallic islands, extended (bi)polaronic states, etc.). The present study provides additional boundary conditions on such refined models, although complete testing would require input from other experiments, such as optical spectroscopy, which probe the charge transport on shorter length and time scales.

We would like to thank B. Verberg of Leiden University, M. de Jongh and L. J. van IJzendoorn of the Technical University of Eindhoven, and H. Schoo of TNO Eindhoven for their participation in part of this work and FOM-NWO and DPI for financial support.
${ }^{1}$ A.J. Heeger, S. Kivelson, J.R. Schrieffer, and W-P. Su, Rev. Mod. Phys. 60, 781 (1988).

${ }^{2}$ C.K. Chiang, C.R. Fincher, Jr., Y.W. Park, A.J. Heeger, H. Shirakawa, E.J. Louis, S.C. Gau, and A.G. MacDiarmid, Phys. Rev. Lett. 39, 1098 (1977).

${ }^{3}$ Y. Cao, P. Wang, and R. Qian, Macromol. Chem. Phys. 186, 1093 (1985).

${ }^{4}$ D.M. de Leeuw, Synth. Met. 55-57, 3597 (1993).

${ }^{5}$ R. Menon, C.O. Yoon, D. Moses, and A.J. Heeger, in Handbook of Conducting Polymers, 2nd ed., (Dekker, New York, 1998).

${ }^{6}$ F. Zuo, M. Angelopoulos, A.G. MacDiarmid, and A.J. Epstein, Phys. Rev. B 39, 3570 (1989).

${ }^{7}$ J. Joo, Y.C. Chung, J.-K. Lee, J.K. Hong, W.P. Lee, S.M. Long, A.J. Epstein, H.S. Woo, K.S. Lang, and E.J. Oh, Synth. Met. 84, 831 (1997).

${ }^{8}$ B. Sanjai, A. Raghunathan, T.S. Natarajan, G. Rangarajan, S. Thomas, P.V. Prabhakaran, and S. Venkatachalam, Phys. Rev. B 55, 10734 (1997).

${ }^{9}$ H. Bässler, Phys. Status Solidi B 175, 15 (1993).

${ }^{10}$ L. Zuppiroli, M.N. Bussac, S. Paschen, O. Chauvet, and L. Furro, Phys. Rev. B 50, 5196 (1994).

${ }^{11}$ S.V. Novikov, D.H. Dunlap, V.M. Kenkre, P.E. Parris, and A.V. Vannikov, Phys. Rev. Lett. 81, 4472 (1998).

${ }^{12}$ S.D. Baranovskii, H. Cordes, F. Hensel, and G. Leising, Phys.
Rev. B 62, 7934 (2000).

${ }^{13}$ A.B. Kaiser, Rep. Prog. Phys. 64, 1 (2001).

${ }^{14}$ We investigated the transition from $1 D$ to $3 D$ in J.A. Reedijk et al., Phys. Rev. Lett. 83, 3904 (1999).

${ }^{15}$ Mössbauer experiments indicate that this holds for $\mathrm{FeCl}_{3}$-doped poly(dialkoxy thiophene), see Ref. 15 .

${ }^{16}$ N.F. Mott, Philos. Mag. 19, 835 (1969).

${ }^{17}$ M.C.J.M. Vissenberg and M. Matters, Phys. Rev. B 57, 12964 (1998).

${ }^{18}$ The exponential decay of $\left|\psi^{2}\right|$ outside the delocalized area $1 / \alpha$ is assumed to be $c$ independent.

${ }^{19}$ A. Miller and E. Abrahams, Phys. Rev. 120, 745 (1960).

${ }^{20}$ V. Ambegaokar, B.I. Halperin, and J.S. Langer, Phys. Rev. B 4, 2612 (1971)

${ }^{21}$ See, e.g., L. E. Reichl, A Modern Course in Statistical Physics (Edward Arnold, London, 1997), p. 13.

${ }^{22}$ The analytic solutions obtained by, e.g., Maple or Mathematica, are straightforward, but too long for showing.

${ }^{23}$ H. Böttger and V. V. Bryksin, Hopping Conduction in Solids (Akademie Verlag, Berlin, 1985).

${ }^{24}$ H.C.F. Martens, P.W.M. Blom, and H.F.M. Schoo, Phys. Rev. B 61, 7489 (2000).

${ }^{25}$ H.C.F. Martens, O. Hilt, H.B. Brom, P.W.M. Blom, J.N. Huiberts, and H.F.M. Schoo, Phys. Rev. Lett. 87, 086601 (2001). 